\title{
SOFRIMENTO NO TRABALHO DE ENFERMAGEM: REFLEXOS DO "DISCURSO VAZIO" NO ACOLHIMENTO COM CLASSIFICAÇÃO DE RISCO
}

\author{
Suffering in the nursing work: reflexes of "empty speech" at the embracement with \\ risk classification \\ Sufrimiento en el trabajo de enfermería: reflejos de lo "discurso vacío" al sector de \\ acogida con clasificación de riesgo
}

Daiane Dal Pai

Liana Lautert ${ }^{2}$

\section{RESUMO}

Estudo que abordou o contexto laboral de trabalhadores de enfermagem que atuam no Acolhimento com Classificação de Risco em um pronto-socorro público de Porto Alegre, Rio Grande do Sul, com objetivo de conhecer suas vivências acerca desta tecnologia para o atendimento em emergência. Pesquisa qualitativa que utilizou como fonte de informações os registros institucionais, cenas de atendimentos observadas e registradas em diário de campo e entrevistas semiestruturadas com os profissionais de enfermagem. As informações foram submetidas à análise de conteúdo, e os dados revelam as características do serviço e os sentimentos da equipe de enfermagem, que apontaram a presença do sofrimento no trabalho ao perceberem seu discurso como "vazio", ou desprovido de sentido. Os achados evidenciaram a necessidade de atenção aos trabalhadores a fim de protegê-los do possível adoecimento e de sofrimento no trabalho, e convocá-los à cogestão desta tecnologia.

Palavras-chave: Acolhimento. Estresse Psicológico. Condições de Trabalho. Enfermagem em Emergência.

\begin{abstract}
This study approached the workplace of nursing workers who work in Embracement with Risk Classification in a public emergency service in Porto Alegre, Rio Grande do Sul, with the objective of learning their experiences about this technology for the emergency care. It is a qualitative research that utilized as information source institutional records, attendance scenes that were observed and registered in a diary field, and semi-structured interviews with the nursing professionals. The information was submitted to content analysis and the results reveal the characteristics of the service and the feelings of the nursing staff that point out the presence of pain at the work when perceiving that their speech is "empty" and void of meaning. The findings evidence the need of giving attention to the workers in order to protect them from sickness deriving from the suffering experience in the work and invite them to comanagement of this technology.
\end{abstract}

Keywords: Embracement. Stress, Psychological. Working conditions. Emergency nursing.

\section{Resumen}

Estudio que abordó el contexto laboral de trabajadores de enfermería que actúan en el sector de Acogida con Clasificación de Riesgo en un servicio de urgencia público en Porto Alegre, Rio Grande do Sul, con el objetivo de conocer sus experiencias acerca de esta tecnología para la atención de urgencia. Se trata de una pesquisa cualitativa que utilizó como fuente de informaciones registros institucionales, escenas de atendimientos observadas y registradas en diario de campo; y entrevistas seme estructuradas con los profesionales de enfermería. Las informaciones fueron sometidas al análisis de contenido y los resultados revelan las características del servicio y los sentimientos del equipo de enfermería, apuntando para la presencia de sufrimiento en el trabajo al percibir su discurso como siendo "vacío" o desproveído de sentido. Los resultados evidencian la necesidad de atención a los trabajadores a fin de protegerlos de enfermedades susceptibles de originarse de la experiencia de sufrimiento en el trabajo y les invitamos a participar en la administración de esta tecnología.

Palabras clave: Acogimiento. Estrés Psicológico. Condiciones de trabajo. Enfermería de urgencia.

\footnotetext{
${ }_{1}^{1}$ Professora Assistente do Departamento de Enfermagem da Faculdade de Enfermagem da Universidade Federal de Pelotas (UFPel). Mestre em Enfermagem. Doutoranda do Programa de Pós-Graduação em Enfermagem da Escola de Enfermagem da Universidade Federal do Rio Grande do Sul (PPGEnf/UFRGS). Porto Alegre- RS. Brasil. E-mail: daiadalpai@yahoo.com.br,2Professora Adjunta do Departamento de Enfermagem Médico-Cirúrgica da Escola de Enfermagem da Universidade Federal do Rio Grande do Sul e do Programa de Pós-Graduação em Enfermagem (PPGEnf/UFRGS). Doutora em Psicologia. Porto Alegre-RS. Brasil. E-mail: lila@enf.ufrgs.br
} 


\section{INTRODUÇÃO}

As diretrizes norteadoras do Sistema Único de Saúde (SUS) apontam a resolutividade como um dos principais desafios a serem enfrentados na consolidação do sistema e na promoção da saúde da população. No entanto, por vezes, a resolutividade tem sido ofertada a partir de uma perspectiva "queixaconduta", como frequentemente se observa nos atendimentos oferecidos pelos superlotados prontos-socorros públicos. Embora muitos avanços já tenham sido alcançados na proposta de reorganizar o modelo de atenção, o pronto-socorro ainda representa um recurso célere para o acesso da população à rede de atenção em saúde, uma vez que os serviços de atenção básica não têm sido suficientes e eficazes no atendimento à saúde da população.

A pressão exercida pela superlotação que caracteriza os serviços públicos de pronto-socorro promove a assistência hospitalar fragmentada, caracterizada em sua maioria por ações específicas e imediatistas, muitas vezes desarticuladas do cuidado integral em saúde. Essas práticas, além de avultarem os gastos, acarretam a descontinuidade da assistência e agravam as situações de doença, principalmente aquelas crônicas que poderiam ser acompanhadas na rede básica, mas acabam nas filas dos serviços de emergência. ${ }^{1}$

Essas situações contribuem para a superlotação dos prontos-socorros, o que resulta em conflitos e insatisfação para os trabalhadores de saúde. Esse sentimento decorre do convivio com a sobrecarga de trabalho e relações tensionadas com colegas e usuários. ${ }^{2} \mathrm{~A}$ insatisfação também atinge os usuários, que permanecem em longas filas, recebem atendimentos em corredores, estabelecem uma relação desumana e até desrespeitosa com os trabalhadores, evidenciando o descumprimento ao direito constitucional de atenção à saúde. Esses problemas merecem atenção uma vez que a receptividade e o respeito são considerados pelos usuários como fundamentais para uma avaliação positiva do acolhimento nos serviços de saúde. ${ }^{3}$

Diante da realidade dos prontos-socorros públicos brasileiros, a Política Nacional de Humanização (PNH) vislumbrou ampliar o acesso e reduzir as filas e tempo de espera propondo o acolhimento com classificação de risco como uma intervenção potencialmente decisiva na reorganização do fluxo de atendimentos na rede e na implementação da promoção da saúde. Essa política visa extrapolar o espaço de gestão local para se afirmar no cotidiano das práticas em saúde, na coexistência das macro e micropolíticas. ${ }^{4}$

Com a PNH, os serviços públicos de pronto-socorro têm implementado estratégias de acesso e acolhimento, as quais podem ser reconhecidas como tentativas de construção de práticas integrais ${ }^{5}$ que requerem 0 uso de critérios de avaliação de risco de forma a classificar as diferentes especificidades das pessoas que procuram este serviço de acordo com o potencial agravo à saúde e, ainda, referenciar aos demais níveis de atenção. ${ }^{4}$

Todavia, por vezes, a noção de acolhimento tem sido limitada a uma atitude profissional de bondade e favor; ou ainda se traduz em recepção administrativa, ambiente confortável e ação de triagem. No entanto, entende-se acolhimento como a adoção de uma postura de aproximação e responsabilização durante o desenvolvimento das ações de atenção e gestão, favorecendo a confiança e o compromisso entre usuários, equipes e serviços. Cabe referir também que a triagem se refere a uma ação excludente a partir da seleção daqueles que serão atendidos, enquanto a classificação de risco se destina à ordenação do fluxo a partir do acolhimento de todos que procuram o pronto-socorro. ${ }^{4}$

Assim, a classificação de risco visa subsidiar a ordem dos atendimentos para que os usuários não sejam orientados pela hora de chegada, mas por parâmetros clínicos de gravidade que permitam identificar as situações que não podem aguardar 0 atendimento devido ao risco de morte. Trata-se, portanto, de uma tecnologia que procura garantir 0 atendimento imediato do usuário que enfrenta risco à vida, além de prever e informar o tempo de espera para os indivíduos que não apresentam este risco.

Para tanto, trabalhadores e instituições precisam responsabilizar-se pela busca de uma relação acolhedora e humanizada, a qual exige metodologias participativas, que considerem a negociação permanente de conflitos na convivência diária nos serviços de saúde. ${ }^{6}$ Compreende-se por humanização a valorização dos diferentes sujeitos implicados no processo de produção de saúde, ${ }^{4}$ por isso, questiona-se a visibilidade dada às necessidades do profissional de saúde, que experimenta acolher classificando o risco em meio a um ambiente complexo em sua natureza e que, além disso, tem sido cercado de demandas que não se referem à finalidade do trabalho nos serviços de urgência e emergência ${ }^{7}$, o que o tem tornado um espaço tensionador das relações humanas.

Visando discutir tal questão, o presente estudo aborda o contexto laboral de trabalhadores de enfermagem que atuam no Acolhimento com Classificação de Risco em um pronto-socorro público de Porto Alegre, Rio Grande do Sul, com objetivo de conhecer as vivências dos profissionais diante desta tecnologia para o atendimento em emergência. Trata-se de investigação integrante da pesquisa que originou a dissertação de mestrado intitulada "Enfermagem, Trabalho e Saúde: cenas e atores de um serviço público de pronto-socorro"s a qual permitiu constatar vivências de sofrimento dos trabalhadores de enfermagem que experimentam o desafio de reorganizar o processo de trabalho.

\section{CAMINHO METODOLÓGICO}

Este estudo tem abordagem qualitativa e descriva e foi realizado em um pronto-socorro de Porto Alegre-RS. A coleta dos dados foi feita, inicialmente, por meio da seleção e análise de documentos institucionais que possibilitaram verificar 
registros acerca dos atendimentos e fluxos do serviço. Da posse desses dados, fez-se observações não participantes por amostragem de tempo, totalizando 14 períodos de observação registradas em diário de campo, com duração de duas horas cada.

Depois, foram realizadas entrevistas semiestruturadas, gravadas em áudio, com 12 trabalhadores da equipe de enfermagem, sendo três enfermeiros e 9 técnicos/auxiliares de enfermagem. Esses sujeitos foram definidos intencionalmente durante os períodos de observação, quando foi possivel identificar os melhores informantes tendo em vista os objetivos da pesquisa. A saturação dos dados foi o critério utilizado para definir o total de participantes submetidos à entrevista.

A coleta dos dados teve duração de 6 meses após a aprovação do Comitê de Ética em Pesquisa da Instituição na qual a investigação foi realizada (AC CP N.022/2005). Foram utilizados Termos de Consentimento Livre e Esclarecido para assegurar os direitos dos participantes, conforme as diretrizes e normas regulamentadoras de pesquisa com seres humanos e a lei dos direitos autorais. Também foi apresentado à instituição sediadora da pesquisa o Termo de Responsabilidade pela Utilização de Dados, a fim assegurar o uso das informações institucionais somente para fins da pesquisa.

Os dados coletados foram submetidos à Análise de Conteúdo ${ }^{9}$, sendo o material examinado minunciosamente a fim de identificar as unidades de registro. Posteriormente, estas foram agrupadas em categorias dando origem aos conjuntos interpretativos, os quais são apresentados e discutidos nos resultados e exemplificados com dados e recortes dos registros institucionais, observações e entrevistas.

\section{APRESENTAÇÃO E DISCUSSÃO DE RESULTADOS}

Os dados encontrados permitiram caracterizar o serviço estudado e as práticas de acolhimento. Assim, discute-se primeiramente o contexto laboral no qual os sujeitos da pesquisa vivenciam o acolhimento com classificação de risco e, em seguida, descrevem-se os sentimentos dos trabalhadores de enfermagem diante do desafio da sua prática.

\section{Características do serviço e o acolhimento}

A análise dos registros de atendimentos dos 12 meses que antecederam a coleta dos dados (total de 188.288 usuários atendidos) mostrou que os casos clínicos representavam o principal motivo $(34,4 \%)$ pela procura do serviço. Esses casos, por sua vez, somaram $57,63 \%$ das causas que levaram ao óbito.

Além do contingente de pessoas que necessitaram dos atendimentos do pronto-socorro somaram-se 6.391 casos de "retorno para consulta" e 1.615 "solicitações externas de exames". Estes dados refletem a desar ticulação do hospital com a rede de saúde e reforçam a sua posição como hegemônica na organização do sistema de saúde.

0 grande número de indivíduos que retornam ao prontosocorro para consulta $(3,4 \%)$ demonstra a falta de um sistema de contrarreferência que integre o usuário do serviço à rede básica, possibilitando-lhe obter um acompanhamento após a ocorrência que o levou ao serviço de emergência. Então, se por um lado há uma atenção básica deficitária, por outro lado também se tem o fomento para o vínculo permanente do usuário ao pronto-socorro, reflexão originada a partir das seguintes fala e observação:

[...] tem os fregueses... esse é freguês, aquele é freguês e lá atrás é freguês. Esta vendo esse senhor? Ele é hipertenso, não deveria estar aqui. Mas eles estão certos, se eu fosse ele também faria isso, ir ao posto de madrugada e não conseguir ficha ou não resolver o problema? Aqui eles são atendidos com certeza e a gente acaba conhecendo eles (Ent).

Uma auxiliar diz à colega, falando de uma senhora que já havia sido atendida: 'Nós vamos ter que ver o social [assistente social] pra ela. Ela não tem como ir embora'. Ouço-as comentando que aquela paciente 'é conhecida na casa'. Ao sair da sala transportada em uma cadeira de rodas, a paciente agradece várias vezes. A auxiliar que afirmava conhecê-la descreve a sua história clínica (Obs).

0 vínculo pressupõe responsabilidade e compromisso profissional com o usuário e sua necessidade. ${ }^{10}$ Dessa forma, percebe-se positivamente o vínculo estabelecido e mencionado pelos trabalhadores; no entanto, sabe-se que a responsabilidade e o compromisso desses profissionais limitam-se ao espaço institucional e ao problema de saúde física, não alcançando a rede de relações sociais e de atenção integral à saúde.

Pode-se ressaltar que o que se vive hoje nos serviços de pronto-socorro não é só influência do aumento dos acidentes de trânsito ou quadros clínicos agudos, mas também do aumento das vítimas da exclusão social, como é o caso dos moradores de rua, que "não têm outras portas para bater". Essa questão é contada pelas profissionais do serviço estudado nas seguintes entrevistas:

[...] vivenciamos bastante a realidade do povo da rua, os moradores de rua, é terrível! Às vezes eles vêm pra cá só para comer e aí nos dá outro problema porque a gente tenta satisfazer, às vezes, o problema da doença deles, que não é um problema de saúde e de doença, e sim um problema de fome (Ent). 
[...] Eles simulam muitos quadros clínicos, principalmente convulsivos para poder ficar [...] porque sabem que aqui têm cama e comida. [...] mas aí tu vai criando um vínculo, amanhã mais ou menos no mesmo horário ele vem de novo, aí tu faz a mesma coisa, aí tu começa ver que ele está vindo para comer ou dormir (Ent).

Conforme o documento final da VIII Conferência Nacional de Saúde, saúde é o resultado das condiç̃̃es de alimentação, habitação, educação, renda, meio-ambiente, trabalho, transporte, emprego, lazer, liberdade e acesso aos serviços de saúde pela população. ${ }^{11}$ Assim, a saúde que a população necessita encontra-se realmente em estado de emergência, mas a resolutividade não é encontrada no pronto-socorro e, conforme diz a profissional de enfermagem:

[...] esse paciente tem essa ilusão de que foi atendido, de que foi tratado [...] a gente ouve relatos que chegam aqui: "Eu já passei em dois, três postos, me mandaram aquil' Sabe, eu não sei até onde vai a verdade ou não, as vezes é tanto disparate. Como um posto [...] pode mandar uma unha encravada e infectada pra cá? Às vezes eu coloco em dúvida a informação recebida. Me custa crer que um posto tenha capacidade de mandar um paciente com esse problema pro HPS. Então é um jogo. É um paciente muitas vezes sem a passagem de ônibus para voltar, aí chega aqui e quer que a gente resolva o problema do universo dele, do mundo dele, então isso é bastante assustador $[\ldots]($ Ent).

Esse relato leva a considerar, ainda, que a rede de serviços de saúde não tem promovido a continuidade de aç̃̃es. Essa problemática tem sido apontada como um dos desafios a serem superados para que os serviços de emergência possam de fato desempenhar o seu papel no atendimento às urgências e emergências e, ainda, para que se rompa com a organização que atribui ao hospital a centralidade nas ações de saúde. ${ }^{1,7}$

Hoje o hospital continua sendo o cume do sistema, e as ações voltadas à cura e centralizadas na figura do médico não contribuem para a reorganização do sistema prevista pelas diretrizes do SUS. Com isso, os próprios hospitais são os mais afetados, pois permanecem pressionados pelos problemas de saúde de grande parcela da população, os quais poderiam ser atendidos por outro tipo de atenção. 0 caminho para superar 0 modelo atual de atenção nos serviços de emergência deverá ser sistêmico e ter o usuário como foco, com redefinição e integração das vocações assistenciais, reorganização de fluxos e repactuação do processo de trabalho. ${ }^{12}$

Nessa direção, muitos são os desafios a serem superados, inclusive na qualidade da atenção à saúde em todos os níveis de atenção. Ou seja, patologias crônicas que deveriam ser passíveis de controle com acompanhamento ambulatorial evoluem para quadros clínicos graves que aumentam gradativamente o risco de morte, o que pode ser observado na descrição de uma situação vivenciada por um profissional de enfermagem:

Eu perguntei:'como é que o senhor veio?' Eu quis saber dele, e ele: 'De ônibus'. A máquina deu H e quando ele estava no posto, a familiar me garantiu que deu 600 ou 800 de glicemia e não tinha médico. Aí eles chamaram um médico, que tomou heroicamente uma caneta e escreveu no boletim: ao HPS, urgente. E me manda de ônibus! Ainda frisou: 'Tu vai rápido porque tu pode entrar em coma'. Como é que isso acontece? De mandar um paciente, naquelas condições? O rapaz estava meio fora do ar, meio torporoso, o raciocínio lento, sabe? E ele tem diabetes há dois anos (Ent).

Dessa forma, casos "não urgentes" se tornam casos graves, e o risco é potencializado pela conduta do profissional que encaminha o paciente a outro serviço sem comprometimento com os possíveis danos à sua saúde no decorrer da busca de atenção. Assim, reproduz-se no imaginário popular a convicção de que é melhor ir direto ao pronto-socorro.

\section{Sofrimento no trabalho: sentimentos e paradoxos da equipe de enfermagem}

No serviço estudado, as práticas de acolhimento com classificação de risco são atribuições de uma equipe formada por técnicos e auxiliares de enfermagem, os quais foram preparados para esta atuação com um treinamento de 80 horas, fornecido pelo Ministério da Saúde. Para dar prioridade aos casos graves, essa equipe realiza a avaliação de risco, que consta de um processo de identificação dos pacientes que necessitam de tratamento imediato, de acordo com o potencial agravo à saúde ou grau de sofrimento. Esta atividade vinha sendo desenvolvida no serviço em pauta há dois anos e foi avaliada positivamente pelos seus protagonistas, conforme pode ser visto no trecho da entrevista:

Através do acolhimento se constata que o paciente
realmente está descompensado e que necessita de
um atendimento de pronto-socorro. Isso veio muito
a calhar para que um paciente com uma glicemia
de 500 não fique esperando por ordem de chegada
como acontecia antes, [...] então, nesse sentido,
também o acolhimento caiu muito bem pra priorizar
o atendimento (Ent).

Por outro lado, o objetivo de reorganizar o fluxo de circulação desordenada dos usuários nas portas do prontosocorro ${ }^{4}$ tem representado um desafio para os auxiliares e 
técnicos de enfermagem responsabilizados por "convencer" os usuários que sua situação não é grave e que necessita de acompanhamento do serviço de atenção básica de saúde. Pois, conforme relatado em entrevista:

[...] a gente aqui no acolhimento tenta, faz aquela pergunta: 'Senhor, e amanhã quando voltar a pressão alta? 0 senhor não pode fazer esse tratamento aqui, aqui não é o lugar, de quatro em quatro horas o médico troca, é outro médico, se ele Ihe prescrever um medicamento, quem é que vai fazer o controle, acompanhar se esse remédio está dando certo ou não?(Ent).

0 acolhimento, como diretriz operacional, deve atender todos os que procuram o serviço, garantindo a universalidade de acesso, acolhendo e escutando os problemas de saúde da população na busca por resolvê-los. Além disso, deve reorganizar o processo de trabalho para que seu eixo central seja uma equipe multiprofissional de acolhimento, bem como qualificar a relação trabalhador-usuário por meio de subsídios humanitários, de solidariedade e cidadania. ${ }^{10}$

A partir desses pressupostos, observa-se que a estrutura do serviço estudado para realizar o acolhimento ainda está longe do ideal. No processo de trabalho observado, 0 acolhimento não acompanha todas as etapas do percurso do usuário e fica restrito a uma equipe de técnicos e auxiliares de enfermagem que, por sua vez, recepcionam o usuário no serviço, antecedendo 0 atendimento clínico, o qual permanece guiado pelas práticas tradicionais, centradas exclusivamente na dimensão biológica e no papel do médico.

Dessa forma, o acolhimento se restringe a uma categoria profissional, em um determinado tempo e espaço físico, o que não atende às propostas do SUS para 0 acolhimento como um modo de operar os processos de trabalho em saúde. ${ }^{4}$ Encontra-se, dessa maneira, a presença da fragmentação das ações de saúde. Cada categoria profissional e cada setor desenvolvem seu próprio objetivo, sem o compromisso de acolher o usuário durante seu percurso no sistema de saúde e concebendo, por vezes, o acolhimento como sinônimo de triagem, concepção similar a dos usuários, conforme apontado por outro estudo ${ }^{6}$ que constatou a compreensão do acolhimento como área física que antecede a convocação para o atendimento.

Também se observou que o pronto-socorro "acolhe" somente no turno do dia e que os antigos processos são mantidos no período da noite. A escassez de recursos humanos é a principal justificativa da equipe de enfermagem para essa organização, sendo que, para o Ministério da Saúde, a quantificação dos atendimentos diários, o perfil da clientela e os horários de pico são considerados para a implementação do acolhimento com classificação de risco. ${ }^{4}$
Além dessa dificuldade, os trabalhadores do acolhimento identificam como obstáculo a responsabilidade de dizer ao usuário que ele deve optar pela atenção básica quando, na verdade, os próprios profissionais desse setor não acreditam que esta seja uma solução.

[...] Dentro desse contexto assustador, a-ssus-tador, não existe uma medicina preventiva, não existe tratamento, as medicações são caras, os postos não têm, [...] então chegam idosos sem tratamento, a PA lá em 200 por 130 porque terminou o remédio. Por mais que tu tentes orientá-los é um discurso vazio porque sai daqui ele vai se deparar com a realidade lá na rua, lá o posto não tem, é uma via crucis, de posto em posto procurando medicação (Ent).

[...] nós não temos... por mais otimista que eu queira ser, nós não temos, nós não podemos, nós não conseguimos vislumbrar uma saída a curto e médio prazo (Ent).

Reconhecendo seu discurso como "vazio", os profissionais afirmam, também, o desconhecimento sobre 0 funcionamento da rede de saúde do Município e os reais limites enfrentados pelos serviços de atenção primária e/ou pelo usuário. Essa limitação requer/exige um programa de capacitação específico para os trabalhadores da saúde sobre o funcionamento da rede pública de assistência e a relação desta com as políticas de saúde. ${ }^{13}$ Neste sentido, acredita-se na validade de um investimento de caráter permanente na formação profissional para o SUS, incluindo todos os profissionais de saúde em discussões que abranjam as dificuldades enfrentadas em todos os níveis da atenção de saúde para identificar as lacunas do sistema e propor medidas com vistas a sua resolução.

Além disso, o que potencializa o discurso "vazio" é a ausência de um sistema de contrarreferência que sustente a orientação dada ao paciente. Dessa forma, o usuário é orientado sobre a necessidade de procurar um serviço de atenção básica, mas não lhe é especificado o serviço, e se o fosse não haveria certeza da resolutividade, o que também contribui para que os objetivos do acolhimento no pronto-socorro não sejam alcançados.

0 projeto de acolhimento requer que o mesmo não seja um ato isolado, mas sim um dispositivo de acionamento de redes internas, externas, multidisciplinares e comprometidas com as respostas às necessidades dos cidadãos. ${ }^{14} \mathrm{Da}$ forma como se observa, ocorre a falta de continuidade do acolhimento na rede de serviços do SUS, o que gera um sentimento de impotência nos profissionais responsáveis pelo acolhimento no pronto-socorro: 
[...] eu tenho que estar centrada ao ponto de saber que eu não vou conseguir mudar isso aqui, então tu fica naquele dilema: compactuar? É desgastante para mim no acolhimento porque toda vez que 0 cliente entra e senta, tu induz a conversa sobre 0 posto, ele se retesa todo, tudo muda, a energia muda, porque aí começa um jogo de pingue-pongue [...] tu não tem argumentos e aí tu quer fazê-lo ver. Tu esta entendendo o dilema que nós vivemos?(Ent)

Diante das situações vivenciadas pela enfermagem para 0 acolhimento, emerge o sentimento de contrariedade, uma vez que, para essas profissionais, as tecnologias leves ganham dimensão de cuidado em $\mathrm{si}^{15} \mathrm{e}$, dessa forma, se confrontam os desejos de um agir justo, ético, com uma exigência institucional a cumprir, o que torna a situação desgastante. Distanciando-se dos princípios éticos, desvia-se o sentido do trabalho, instalando-se um "estado de vida contrariado" 16:125, suscetibilizando ao sofrimento no trabalho.

A lacuna entre o que deveria ser feito e o que vem sendo feito tem implicado diretamente nos sentimentos dos profissionais de enfermagem, os quais se veem obrigados a sustentar um discurso "vazio", do qual nem mesmo eles acreditam. Diante dsso, constata-se uma situação de alerta à saúde dos trabalhadores, uma vez que o sentido do trabalho é constituinte da identidade do trabalhador e, dessa forma, influencia diretamente a saúde dessas pessoas. ${ }^{17}$

0 fazer em saúde carrega o atributo moral da atividade de cuidar das pessoas e/ou salvar vidas, o que é merecedor de reconhecimento da sociedade e dos pares - colegas de trabalho e, por isso, se torna fator benéfico à saúde no trabalho. ${ }^{18}$ No entanto, quando o trabalhador se percebe vencido pela tarefa inalcançável, o fazer se torna desprovido de sentido, de motivação e de luta pela conquista dos desejos individuais e coletivos, assim como da atenção qualificada em saúde.

A realização da classificação de risco isoladamente não garante a melhoria da qualidade da atenção, são necessárias pactuações internas e externas para a viabilização do processo, com a construção de fluxos na rede de atenção em saúde. ${ }^{16}$ Com isso, pode-se afirmar que muito se tem a fazer em prol da reestruturação do processo de trabalho a fim de construir um projeto de acolhimento.

\section{CONSIDERAÇÕES FINAIS}

0 acolhimento com classificação de risco enquanto tecnologia que visa a reorganizar a promoção da saúde no SUS exige investimentos estruturais na rede de atenção em saúde como um todo, pois sem a certeza do acesso por meio da contrarreferência, os usuários acabam por crer que o prontosocorro é de fato a única porta de entrada ao sistema de saúde. Nessa conjuntura, os profissionais de enfermagem que atuam no acolhimento revelaram sofrer, uma vez que compreendem que a sua intervenção junto ao usuário é desprovida de resolutividade e o seu fazer, desprovido de sentido.

Os achados dessa investigação alertam para a atenção à equipe de enfermagem, que atua com a tarefa de acolher $\mathrm{e}$ reorientar o fluxo de atendimentos no pronto-socorro, sob riscos de reforçarem a ineficiência do sistema de referência e contrarreferência em saúde, do ponto de vista institucional, e de causarem dano à própria saúde devido à falta de motivação. Assim, o sofrimento constatado no trabalho de campo pode ser considerado indicador de vulnerabilidade e risco à saúde para as trabalhadoras de enfermagem.

Esta pesquisa oferece subsídios importantes para gestores dos serviços de saúde que pretendem implementar e/ ou qualificar o acolhimento com classificação de risco, uma vez que se observou a necessidade de assistir de forma permanente aos trabalhadores envolvidos nesta atividade para serem cogestores do processo e para que de fato se obtenham avanços na atenção das urgências e emergências. A partir da vivência destes profissionais, também é possível vislumbrar alguns entraves na organização do Sistema, os quais carecem de investimentos para se efetivar e qualificar a rede de atenção à saúde e, em consequência, desafogar os serviços de urgência e emergência superlotados.

Como limitação deste estudo, aponta-se o foco exclusivo dado aos trabalhadores de enfermagem, sendo que outros profissionais da equipe de saúde também experimentam, de alguma forma, as repercussões da inserção do acolhimento com classificação de risco junto ao processo de trabalho no pronto-socorro, o que remete à recomendação de pesquisas com esses trabalhadores.

\section{REFERÊNCIAS}

1 Valentim MRS, Santos MLSC. Políticas de saúde em emergência e a enfermagem. Rev Enferm Uerj. 2009 abr/jun; 17(2): 285-89.

2 Deslandes SF. Frágeis deuses: profissionais de emergência entre os danos da violência e a recriação da vida. Rio de Janeiro: Fiocruz; 2002.

3 Ramos DD, Lima MADS. Acesso e acolhimento aos usuários em uma unidade de saúde em Porto Alegre, Rio Grande do Sul, Brasil. Cad Saude Publica. 2003; 19:27-34.

4 Ministério da Saúde (BR). Núcleo Técnico de Política Nacional de Humanização-PNH. Cartilhas da PNH: acolhimento com classificação de risco. Brasília(DF); 2004.

5 Cecilio LCO, Merhy EE. A Integralidade do cuidado como eixo da gestão hospitalar. In: Pinheiro R, Mattos RA, organizadores. Construção da integralidade: cotidiano, saberes e práticas em saúde. Rio de Janeiro:Uerj/ IMS/Abrasco; 2003. p. 197-210.

6 Souza ECF, Vilar RLA, Rocha NSPD, Uchoa AC, Rocha PM. Acesso e acolhimento na atenção básica: uma análise da percepção dos usuários e profissionais de saúde. Cad Saude Publica. 2008; 24 (supl. 1): 100-10. 
7 Garlet ER, Lima MADS, Santos JLG, Marques GQ. Finalidade do trabalho em urgências e emergências: concepções de profissionais. Rev Latinoam Enfermagem. 2009 jul/ago; 17(4):

8 Dal Pai D. Enfermagem, trabalho e saúde: cenas e atores de um serviço público de pronto socorro. [dissertação]. Porto Alegre: Universidade Federal do Rio Grande do Sul; 2006.

9 Bardin L. Análise de conteúdo. Lisboa: Ed 70;, 2009.

10 Coelho M0, Jorge MSB. Tecnologia das relações como dispositivo do atendimento humanizado na atenção básica à saúde na perspectiva do acesso, do acolhimento e do vínculo. Cienc Saude Colet. 2009 set/out; 14(supl.1):1523-531.

11 Cunha JPP, Cunha RE. Sistema Único de Saúde Princípios. In: Campos FE, Tonon LM, Junior M. Caderno de Saúde. 0 planejamento e gestão em saúde. Belo Horizonte: Coopmed; 2003.

12 Bittencourt RJ, Hortale VAA qualidade nos serviços de emergência de hospitais públicos e algumas consideraç̃os sobre a conjuntura recente no município do Rio de Janeiro. Cienc Saude Colet. 2007; 12(4): 929-34. 13 CostaALRC. As múltiplas formas de violência no trabalho de enfermagem: o cotidiano de trabalho no setor de urgência e emergência clínica em um hospital público. [tese]. Ribeirão Preto: Escola de Enfermagem de Ribeirão Preto , Universidade de São Paulo; 2005.

14 Ministério da Saúde (BR). Secretaria de Atenção à Saúde. Política Nacional de Humanização da Atenção e Gestão do SUS. Acolhimento e classificação de risco nos serviços de urgência. Brasília(DF); 2009.

15 Silva DC, Alvim NAT, Figueiredo PA. Tecnologias leves em saúde e sua relação com o cuidado de enfermagem hospitalar. Esc Anna Nery. 2008; 12(2): 291-98

16 Machado MH. Gestão pública e ética no trabalho. In: Seminário Internacional de Políticas de Recursos Humanos. Brasília(DF); 2002.

17 Lancman S, Sznelwar LI, organizadores. Christophe Dejours: da psicopatologia à psicodinâmica do trabalho. Rio de Janeiro: Fiocruz; 2004.

18 Dal Pai D, Lautert L. 0 trabalho em urgência e emergência e a relação com a saúde das profissionais de enfermagem. Rev Latino-am Enfermagem. 2008; 16(3): 439-44.

\section{NOTA}

aEla se refere ao glicosímetro. HI é o resultado do teste, quando os níveis de glicemia estão superiores ao que o aparelho reconhece. 